\title{
Classification Manual for Voice Disorders-I - CMVD-I
}

\author{
Comentado por: Mara Behlau ${ }^{1}$, Gisele Gasparini ${ }^{2}$
}

Verdolini K, Rosen CA, Branski RC, editors. Classification Manual for Voice Visorders-I. Special Interest Division 3, Voice and Voice Disorders. Rockville: ASHA; 2006.

O Manual de Classificação dos Distúrbios Vocais CMVD-I é um esforço sistêmico de organizar a literatura das variadas alterações que podem produzir problemas vocais e criar uma referencia padrão, tendo como público-alvo profissionais de diversas formações. $\mathrm{O}$ manual pretende ainda oferecer uma perspectiva para classificar os distúrbios vocais e facilitar a comunicação entre os profissionais. Cada uma das entradas deste manual contém uma descrição da condição ou lesão, distinguindo as características essenciais das associadas, com a descrição do impacto vocal produzido, aspectos demográficos dos pacientes, curso clínico, fatores desencadeantes, além de dados sobre diagnóstico diferencial e critérios de classificação.

A versão atual é o progresso de um esforço iniciado em 1995 pela ASHA sob a direção da Profa. Dra. Christy Ludlow. Desde então, criou-se um trabalho continuado para se produzir a primeira referência deste tipo na área de voz. Assim, os editores são Verdolini K, Rosen C e Branski R, e os autores colaboradores, em ordem alfabética, são: Andrews M, Bless D, Boone D, Casper J, Glaze L, Karnell M, Ludlow C e Stemple J, com contribuições adicionais de BarkmeierKraemer J, Duffy J, Leonard R, Shrivastav R e Thibeault S.

O CMVD-I não se propõe a ser um guia de diagnóstico e tratamento e sim um esforço puramente descritivo. A contribuição extrapola a classificação das alterações laríngeas que geralmente se mantêm nos limites do órgão e inclui as alterações vocais sistêmicas ou extra-laríngeas, como as doenças neurológicas e psicológicas. A proposta do texto é de ser a base para edições futuras, o que aparece na designação "I", após o título. A grande contribuição é a enorme organização apresentada e a abertura para uma discussão sobre nomenclatura na área. O CMVD é publicado sob patrocínio do SID3 da ASHA, que convidou o Dr. Clark Rosen para representar a contribuição médica.

(1) Coordenadora e Docente do Curso de Especialização em Voz do Centro de Estudos da Voz - CEV - São Paulo (SP), Brasil; Doutora em Ciências dos Distúrbios da Comunicação Humana pela Universidade Federal de São Paulo - UNIFESP-EPM - São Paulo (SP), Brasil.

(2) Vice-coordenadora e Docente do Curso de Especialização em Voz do Centro de Estudos da Voz - CEV - São Paulo (SP), Brasil; Mestre em Ciências dos Distúrbios da Comunicação Humana pela Universidade Federal de São Paulo - UNIFESP-EPM - São Paulo (SP), Brasil.

Endereço para correspondência: Centro de Estudos da Voz - CEV. R. Machado Bittencourt, 361, $10^{\circ}$ andar, São Paulo - SP, CEP 04044-001. Email: giselegasparini@uol.com.br
É importante ressaltar a distinção entre diagnóstico e a classificação: o diagnóstico refere-se a um rótulo que um profissional qualificado aplica, indicando a presença de uma certa condição médica ou uma condição de outra natureza; já a classificação de um distúrbio vocal, no presente manual, refere-se à atribuição de um problema de voz como pertencente a uma categoria específica de distúrbios. Classificar requer que se considere o tipo de condição médica ou outras condições que possam contribuir para o problema de voz, assim como os atributos da função vocal que são afetados por tais condições. Quando um clínico faz um diagnóstico, a classificação do distúrbio pode envolver um ou vários profissionais. Tal manual não está isento de controvérsias sobre a natureza de algumas condições, principalmente pelo dinamismo do avanço nas questões clínicas e da ciência básica, assim como pelos diferentes modelos usados por clínicos. Em tais situações, o manual ressaltou as controvérsias e apresentou pontos de vista alternativos ao conhecimento atual e termos existentes na literatura. Os autores tentaram apresentar o estado da arte quanto às informações relevantes aos distúrbios vocais, algumas das quais desafiam os conceitos tradicionais. A conceitualização do manual foi modelada de modo semelhante ao DSM (Diagnostic and Statistic Manual), publicado pela Associação Psiquiátrica Americana, embora com um perfil menos rígido. O CMVD-I foi adaptado para permitir flexibilidade e, ao contrário da série do DSM, o objetivo não é o diagnóstico e sim a categorização de uma certa condição e das disfunções envolvidas na produção vocal. Além disso, o manual não baseia as classificações em categorias derivadas estatisticamente, como o DSM. O CMVD-I usa cinco dimensões e não os três eixos maiores do DSM (Eixo I - distúrbios clínicos; Eixo II - distúrbios da personalidade e atraso mental e Eixo III - condições médicas gerais).

A dimensão 1 refere-se às categorias das condições que podem contribuir para um problema de voz. Tais condições incluem: alterações estruturais, inflamações, trauma ou lesão, doenças sistêmicas, distúrbios aero-digestivos não laríngeos, transtornos psiquiátricos, distúrbios neurológicos e condições não especificadas de outra forma. Essa última categoria é utilizada para os casos que não preenchem os critérios de classificação descritos nesse texto: pode ser que o clínico seja incapaz de determinar a causa da disfonia, ou que os critérios de classificação não sejam contemplados em sua totalidade, ou que haja pendências quanto à suspeita 
diagnóstica. Assim, essa categoria pode ser utilizada para pacientes com queixas de alterações vocais consistentes ou presença de disfonia que não pode ser explicada por uma condição médica ou uso de substância e que não contemplam os critérios das outras condições descritas no texto. Em cada uma dessas classes gerais de condições, o manual oferece informação sobre: as características essenciais que definem a condição; as características associadas que podem acompanhar a condição, mas não são essenciais na sua descrição; as limitações vocais associadas a esta condição (dados acústicos, aerodinâmicos e imagem laríngea, quando disponível); idade do início da alteração; curso (a progressão típica da condição); complicações ou seqüelas da condição ou de seus tratamentos; fatores de predisposição ou fatores de risco; frequiência considerando-se a população como um todo; proporção por sexo; padrão familiar; diagnóstico diferencial com informações sobre as outras condições que devem ser comparadas; e, finalmente, critérios de classificação, que são os dados necessários para identificar a condição em si e atribuir um problema de voz a esta condição específica, compreendendo-se que o nexo causal entre uma condição diagnóstica e um problema vocal nem sempre é possível, pois quando o paciente é avaliado, o problema de voz já se desenvolveu e vemos somente o resultado da evolução deste distúrbio.

A dimensão 2 refere-se ao nível de certeza da classificação, o que reflete a confiança relativa sobre a condição presente e a atribuição do problema de voz a esta condição especificada. Sugere-se utilizar tanto para as condições médicas quanto para os atributos vocais a seguinte classificação:

A: Classificação provisória - quando a informação é insuficiente e a classificação futura só poderá ser feita na presença de outros sinais e sintomas.

B: Classificação postergada - quando a informação é insuficiente e a classificação futura depende do curso clínico ou da resposta a um tratamento.

C: Classificação provisória e dependendo do diagnóstico realizado por um profissional apropriado - há informação suficiente para fazer uma classificação em processo, contudo há uma certa incerteza (ex. Classificação provisória de Lupus até confirmação de análise de sangue).

D: Boa certeza da acurácia na classificação.

E: Certeza da acurácia na classificação.

A dimensão 3 codifica a severidade da condição atual em termos de severidade médica e vocal. Assim a avaliação da severidade atual tem dois aspectos, sendo o primeiro o reflexo da gravidade da doença ou condição subjacente (ex. DPOC) e o segundo relacionado à severidade do problema vocal. A severidade do problema vocal deve refletir a integração dos sinais e sintomas, assim como da qualidade de vida em relação à voz. Pode-se usar esquema geral de quatro pontos, tanto para a severidade da condição médica como para a vocal:

Normal: sem sinais, sintomas ou limitações funcionais.

Discreta: sinais, sintomas e limitações funcionais discretas. Moderada: sinais, sintomas e limitações funcionais entre discretas e acentuadas.
Acentuada: sinais, sintomas e limitações funcionais significantes.

A dimensão 4 codifica a percepção do paciente sobre o impacto de seu funcionamento vocal na qualidade de vida. Para tanto são usados questionários como instrumentos que refletem a percepção dos pacientes sobre o problema. Os dois protocolos exemplificados no manual são o IDV e o QVV.

A dimensão 5 codifica as informações sobre o curso clínico da condição médica ou de outras condições e, em separado, o curso da função vocal. O curso clínico pode ser semelhante em uma condição específica, assim como suas características associadas. Entretanto, em outros casos, o curso pode diferir. Consideramos cinco possibilidades:

R: Em Resolução - usado quando as condições médicas ou outras condições estiveram presentes no passado, mas não no momento atual, como em casos de nódulos.

M: Em Melhoria - esse verbete indica que a condição médica ou vocal esta melhorando de modo geral.

E: Estável - essa designação indica que a condição médica ou vocal está geralmente estável, nem melhorando, nem deteriorando e nem em recorrência cíclica.

RC: Recorrência cíclica - essa designação indica um curso clínico com recorrências intermitentes.

D: Em Deterioração - essa designação indica um curso clínico geral que está em declínio.

Veja, a seguir, a classificação dos distúrbios vocais proposta no CMVD-I. Com certeza, um grande avanço foi oferecido à área e as discussões e controvérsias advindas dessa proposta contribuirão para o avanço da caracterização dos problemas vocais. O esforço é único e nunca antes se chegou a uma clareza tão grande na área, apesar das limitações inerentes a toda e qualquer proposta de taxonomia.

\section{CLASSIFICAÇÃO DOS DISTÚRBIOS VOCAIS: CMVD-I}

\section{DOENÇAS ESTRUTURAIS DA LARINGE}

1100. Lesões Laríngeas Malignas 1110. Lesões Malignas das Pregas Vocais 1120. Displasia/carcinoma in situ

1130. Afecção Maligna da Laringe (origem fora das pregas vocais)

1200. Anormalidades do Epitélio e Lâmina Própria das Pregas Vocais

1210. Lesões Benignas Focais da Lâmina Própria

1211. Nódulos de Prega Vocal

1212. Massa fibrosa - Subepitelial

1213. Massa fibrosa - Ligamento

1214. Pólipo(s) de Prega Vocal

1215. Cisto de prega vocal - Subepitelial

1216. Cisto de prega vocal - Ligamento

1217. Lesão Reativa de Prega Vocal

1220. Edema de Reinke

1230. Cicatriz de Prega Vocal

1231. Cicatriz de Prega Vocal Propriamente Dita

1232. Sulco de Prega Vocal 
1240. Granuloma de Prega Vocal

1241. Granuloma de Prega Vocal não relacionado a Intubação

1242. Granuloma de Prega Vocal Relacionado a Intubação/Úlcera de Contato

1250. Queratose (Também Descrita como Leucoplasia ou Eritroplasia)

1260. Papilomatose Respiratória Recorrente (PRR)

1270. Estenose Subglótica

1280. Estenose Adquirida Glótica /Laríngea (Membrana glótica anterior)

1300. Anormalidades Vasculares das Pregas Vocais

1310. Hemorragia de Prega Vocal

1320. Varizes e Ectasia de Prega Vocal

1400. Alterações Congênitas e de Maturação que Afetam a Voz

1410. Membranas Congênitas (Sinéquia)

1420. Síndrome do Choro do Gato

1430. Laringomalácia

1440. Puberfonia

1450. Presbifonia

2000. INFLAMAÇÕES LARÍNGEAS

2100. Artrite Cricoaritenóidea e Cricotireóidea

2200. Laringite Aguda

2300. Refluxo Laringofaríngeo

2400. Sensibilidade Química - Síndrome da Laringe Irritável

3000. TRAUMA OU LESÃO DA LARINGE

3100. Trauma Laríngeo Interno

3110. Trauma da Mucosa Laríngea (Químico ou Térmico)

3120. Lesões da Mucosa Laríngea por Intubação/ Extubação

3130. Deslocamento da Aritenóidea

3200. Trauma Laríngeo Externo

4000. CONDIÇÕES SISTÊMICAS QUE AFETAM A VOZ

4100. Endócrinas

4110. Hipotireoidismo

4120. Hipertireoidismo

4130. Desequilíbrio dos hormônios sexuais

4140. Anormalidades do Hormônio de Crescimento (Hiperpituitarismo)

4200. Imunológicas

4210. Doenças Alérgicas do Trato Respiratório Superior

4220. HIV e SIDA

4230. Síndrome da Fadiga Crônica

4240. Lupus Eritomatoso Sistêmico

4250. Síndrome de Sjogren

4260. Escleroderma

4270. Doença de Wegener

4300. Alterações Músculo-Esqueléticas que Afetam a Voz

4310. Trauma por Uso Excessivo e Trauma por Esforço Repetitivo
4320. Fibromialgia

4330. Síndrome de Ehler Danlos

4400. Desidratação

5000. DISTÚRBIOS AERODIGESTIVOS NÃOLARINGEOS QUE AFETAM A VOZ

5100. Doenças Respiratórias que Afetam a Voz

5110. Asma

5120. Doença Pulmonar Obstrutiva Crônica - DPOC

5200. Gástricas

5210. Doença do Refluxo Gastroesofágico

5300. Doença Infecciosa do Trato Aéro-digestivo

5305. Laringotraqueobronquite (Crupe)

5310. Coqueluche

5315. Difteria

5320. Pneumonia

5325. Sinusite Infecciosa

5330. Tuberculose

5335. Infecção das Vias Aéreas Superiores - IVAS

5340. Epiglotite Aguda

5345. Sífilis

5350. Sarcoidose

5355. Escleroma da Laringe (Klebsiella Rhinoscleromatis)

5360. Lepra (que afeta a laringe)

5365. Actinomicose

5400. Infecções Micóticas (Fúngicas)

5410. Blastomicose

5420. Histoplasmose

5430. Candidíase

5440. Coccidioidomicose (Febre de Valley, Febre do Deserto, Febre de São Joaquim)

6000. TRANSTORNOS PSIQUIÁTRICOS E PSICOLÓGICOS QUE AFETAM A VOZ

6010. Transtornos Somatoformes

6011. Transtornos de Somatização

6012. Transtornos de Conversão

6013. Transtornos de Dor

6014. Hipocondriase

6020. Distúrbio Factício

6030. Mutismo Seletivo

6040. Ansiedade

6041. Distúrbio do Estresse Pós-traumático

6042. Distúrbio de Ansiedade Generalizada

6050. Transtornos de Humor

6051. Trasntorno Depressivo Maior (Recorrente)

6052. Transtorno Bipolar I

6060. Transtono da Identidade de Gênero

6070. Polidipsia Psicogênica

6080. Flutuações Vocais Psicogênicas - semelhante a Tremor

7000. DISTÚRBIOS NEUROLÓGICOS QUE AFETAM A VOZ

7100. Lesão do Sistema Nervoso Periférico

7110. Lesão do Nervo Laríngeo Superior 
7120. Paralisia Unilateral do Nervo Laríngeo Recorrente

7130. Paresia Unilateral ou Bilateral do Nervo Laríngeo Recorrente

7140. Paralisia Bilateral do Nervo Laríngeo Recorrente - Periférica

7150. Miastenia Gravis

7160. Neuropatia Periférica (Neuropatia, Charcot Marie Tooth, ou neuropatia hereditária sensório-motora).

7170. Tremor Fisiológico Acentuado que Afeta a Voz

7200. Distúrbios de Movimento que Afetam a Laringe

7210. Disfonia Espasmódica Adutora

7220. Disfonia Espasmódica Abdutora

7230. Disfonia Espasmódica Mista (adutora e abdutora)

7240. Tremor Distônico que Afeta a Voz

7250. Tremor Essencial que Afeta a Voz

7260. Síndrome de Meige (Distonia Orofacial)

7270. Estereotipias Tardias (Discinesia Tardia)

7280. Síndrome de Gilles de La Tourrett

7300. Distúrbio do Sistema Nervoso Central

7305. Esclerose Lateral Amiotrófica (ELA; Doença de Lou Gehrig)

7310. Síndrome de Wallenberg (Síndrome Medular Lateral/Infarto)
7315. Doença de Parkinson

7320. Atrofia de Múltiplos Sistemas (Síndrome de Shy-Drager, Degeneração Estriatonigral, Atrofia Olivopontocerebelar Esporádica)

7325. Paralisia Supranuclear Progressiva (Inclui Paralisia Pseudobulbar e Síndrome de SteeleRichardson-Olszewski)

7330. Esclerose Múltipla

7335. Distúrbio Cerebelar

7340. Coréia de Huntington

7345. Paralisia Central Bilateral do Nervo Laríngeo Recorrente

7350. Mioclonus

8000. OUTROS DISTÚRBIOS QUE AFETAM A VOZ

8010. Disfonia por Tensão Muscular (Primária)

8020. Tensão Muscular/Disfonia Adaptativa (Secundária)

8030. Disfonia Vestibular

8040. Distúrbio do Movimento Paradoxal das Pregas Vocais (Disfunção das Pregas Vocais)

9000. DISTÚRBIOS VOCAIS: NÃO DIAGNOSTICADOS OU DE CAUSA NÃO ESPECIFICADA DE OUTRA FORMA 